\title{
The Influence of Rearing Conditions on the Physical Growth of Captive Japanese Macaques (Macaca fuscata)
}

\author{
Juri SUZUKI ${ }^{1}$, Norikatsu MIWA ${ }^{1)}$, Kiyonori KUMAZAKI ${ }^{1)}$, Masamitsu ABE ${ }^{1)}$, Yoshiro KAMANAKA ${ }^{1}$, \\ Nobuko MATSUBAYASHI ${ }^{1)}$, Shunji GOTOH $^{1)}$ and Kiyoaki MATSUBAYASHI ${ }^{1)}$ \\ ${ }^{1)}$ Center for Human Evolution Modeling Research, Primate Research Institute, Kyoto University, Inuyama, Aichi 484-8506, Japan
}

(Received 4 August 2000/Accepted 5 December 2000)

ABSTRACT. To clarify the influence of rearing conditions on the growth of various body parts of Japanese macaques (Macaca fuscata), two groups reared under different conditions, i.e., a group born and reared in open enclosures (Enclosure group) and another consisting of macaques born and reared in cages (Caged group), were somatometrically analyzed. Somatometric data on 36 measures of various body parts were collected from 77 males and 92 females. Growth in many body parts was smaller in the Caged group than in the Enclosure group. Body parts that exhibited large incremental increases were more sensitive to differences in rearing space at the infantile growth stage in both sexes. Recovery from delayed growth at the pubertal growth stage was found in many body parts. However, the size of some locomotor elements such as the wrist and hand, and ankle and foot strongly reflected limitations of space and changes due to this were irreversible. Females were more sensitive than males to such differences in rearing conditions. We conclude that open enclosures with ample rearing space are necessary for the innate growth of Japanese macaques to occur.

KEY WORDS: Japanese macaque, physical growth, rearing condition, somatometry.

J. Vet. Med. Sci. 63(4): 361-366, 2001

The Japanese macaque is routinely used for experiments in various fields such as medicine and biology because of its phylogenetic closeness to human beings. Although investigators have attempted to elucidate the morphological characteristics of Japanese macaques, their main focus has not been the morphological differences caused by various environmental factors, and discussion has been limited to the influences of nutrition, climate and genetic background [1$6,16]$. In many laboratories, non-human primates, including Japanese macaques, are reared in cages. Although growth is thought to be suppressed in cages because of their limited space, a detailed comparison of differences in growth between caged Japanese macaques and free-ranging ones has not been carried out to date. Japanese macaques are reared in both open enclosures and cages for breeding purposes at the Primate Research Institute of Kyoto University. As these two rearing systems are quite different, especially in terms of space, the purpose of the present study was to clarify what morphological characteristics are subject to changes in growth in Japanese macaques reared in a limited space environment.

\section{MATERIALS AND METHODS}

This study was approved by the Animal Welfare and Animal Care Committee of the Primate Research Institute of Kyoto University.

Animals: All Japanese macaques used in this study were born and reared at the Primate Research Institute of Kyoto University and were nursed by their own mothers. They were classified into two groups according to their rearing conditions. One group consisted of macaques that were born and grew up in open enclosures. The other group con- sisted of macaques that were born in individual cages, were separated from their mothers after 1 year of age, and subsequently grew up in the same kind of individual cage or were housed in outdoor cages. These two groups were named the Enclosure group and the Caged group, respectively.

Rearing conditions : Areas of the corral enclosures, outdoor cages, and individual cages were 496 to $2,100 \mathrm{~m}^{2}, 9$ $\mathrm{m}^{2}$, and 0.5 to $0.6 \mathrm{~m}^{2}$, respectively. Corral enclosures were surrounded by a reinforced concrete $3.5 \mathrm{~m}$ wall and $4 \mathrm{~m}$ high climbing frames were built in the center. Outdoor and individual cages were constructed of stainless steel frames 2.2 $\mathrm{m}$, and 0.8 to $0.85 \mathrm{~m}$ high, respectively. Macaques moved and climbed on frames freely within the enclosures. Due to restrictions of space, however, they could move much less freely in outdoor or individual cages. Individually caged macaques were kept in a room with a temperature range between $10^{\circ}$ to $25^{\circ} \mathrm{C}$ and a lighting schedule of $12 \mathrm{~L}-12 \mathrm{D}$ (lights on at 06:00). All macaques were fed every day with commercial monkey-chow (Oriental Yeast Co., Ltd., Tokyo, Japan) supplemented with sweet potatoes three times a week. They took water ad libitum.

Somatometry : Somatometric data on Enclosure group macaques were obtained by measuring 85 individuals from 1 through to 7 years of age. Data on Caged group macaques were obtained by measuring 10-14 individuals annually from 1 to 7 years of age. The Caged group macaques were separated into 2 subgroups, macaques in individual cages and those in outdoor cages, from 2 years onward. The number of individuals from the Enclosure and Caged groups subjected to somatometric analyses at each stage are shown in Table 1.

Macaques were anesthetized by an intramuscular injection of ketamine-hydrochloride (Sankyo, Co., Ltd., Tokyo, 
Table 1. Number of macaques subjected to somatometric measurements at various ages

\begin{tabular}{|c|c|c|c|c|c|c|}
\hline \multirow[b]{4}{*}{ Age (year) } & \multicolumn{6}{|c|}{ Number of macaques } \\
\hline & \multicolumn{4}{|c|}{ Born in cage $\mathrm{e}^{\mathrm{a})}$} & \multirow{2}{*}{\multicolumn{2}{|c|}{$\begin{array}{l}\text { Born in enclosure }^{\text {b) }} \\
\text { Reared in enclosure }\end{array}$}} \\
\hline & \multicolumn{2}{|c|}{ Reared in individual cage } & \multicolumn{2}{|c|}{ Reared in outdoor cage } & & \\
\hline & Male & Female & Male & Female & Male & Female \\
\hline 1 & 7 & 7 & & & 9 & 10 \\
\hline 2 & 3 & 3 & 3 & 3 & 7 & 10 \\
\hline 3 & 3 & 3 & 3 & 3 & 5 & 7 \\
\hline 4 & 3 & 3 & 3 & 3 & 4 & 7 \\
\hline 5 & 3 & 3 & 3 & 3 & 2 & 7 \\
\hline 6 & 3 & 3 & 3 & 3 & 4 & 4 \\
\hline 7 & 3 & 2 & 3 & 2 & 3 & 6 \\
\hline Total & 25 & 24 & 18 & 17 & 34 & 51 \\
\hline
\end{tabular}

a) Fourteen individuals were subjected to somatometric analyses at each stage. Half of the individually caged macaques were transferred to outdoor cages at 1 year of age. Since some macaques died due to accidents, the summed numbers decreased between the ages of 2 and 7 years.b) All macaques were different individuals.

Japan), $10 \mathrm{mg} / \mathrm{kg}$ body weight, and measured with somatometric instruments used for humans. The measurement method of Martin and Saller [7] was applied with some modifications of Hamada [1] and Iwamoto [6]. A two-way ANOVA with two factors, group and age, was performed on each individual measurement. When the difference in a measurement between groups was significant $(\mathrm{p} \leq 0.05)$, the Duncan multiple range test was performed separately for males and females. Although Caged group macaques were further separated into individual and outdoor cages from 2 years of age, data obtained from caged macaques were treated statistically as one group since no significant differences were evident between these subgroups.

\section{RESULTS}

The somatometric data of 36 body part items are summarized in Tables 2 and 3. The timing of major physiological growth events, such as weaning and sexual maturation, were the same for the two groups, and similar to those reported previously in Japanese macaques [8, 10-12]. Trends of growth in the Enclosure group macaques were similar to those of free-ranging Japanese macaques [3, 4]. Growth in many body parts, however, was different between Enclosure and Caged group macaques. In 20 measurements in males and 28 measurements in females, the average values of Caged group macaques were significantly smaller $(\mathrm{p} \leq 0.05)$ than those of the Enclosure-group, from 1 to 7 years of age. Items that exhibited larger measurement values in Caged group macaques occurred five times in males and once in females.

Characteristics of growth in each body region examined were as follows:

Head: The growth of all cranial measurements was statistically the same in males of both the Enclosure and Caged groups, except that head length and height in the Caged group was significantly smaller than that of the Enclosure group at 4 and 7 years of age, respectively. In females, how- ever, most Caged group measurements were significantly smaller than those of the Enclosure group from 3 to 7 years of age. Head length and breadth of Caged group females were also significantly smaller than those of Enclosure group females at 1 year of age.

Face: No difference in the growth of face-related body parts was found between Enclosure and Caged group males with the exceptions of facial height in the Caged group, which was significantly smaller than that in the Enclosure group at 4 years of age, and bigonial breadth which was significantly larger in the Caged group than in the Enclosure group at 5 and 6 years. In females, the growth of bigonial breadth of the Caged group and the Enclosure group was similar, whereas growth in facial height and upper facial height of the Caged group was significantly smaller than those of the Enclosure group from 3 years onward.

Trunk: In males, 4 of 8 trunk related measurements, i.e., sitting height, tail length, anterior trunk length and bi-acromial breadth of the Enclosure and Caged groups showed similar values from 1 year of age onward, except for larger values of sitting height at 6 years, and anterior trunk length at 5 years in the Caged group. Chest breadth and depth of the Caged group were significantly smaller than the Enclosure group at the infantile stage. In contrast, bi-iliac breadth and bitrochanteric breadth of the Caged group were significantly larger at the pubertal stage. In females, 4 measurements exhibited differences between the groups. Three of those, sitting height, chest breadth and depth, were significantly smaller in the Caged group at some growth stages, whereas bi-iliac breadth of the Caged group was significantly larger than that of the Enclosure group at 7 years.

Limbs: In Caged group males, 7 of 12 limb-related measurements, i.e., hand length, hand breadth, 3rd digit length, foot length, foot breadth, 1st toe length and 3rd toe length, were significantly smaller than those of the Enclosure group at some growth stages. In particular, for hand length and breadth, and foot length and breadth, this tendency was remarkable. Only upper arm length of the Caged group was 
Table 2. Summary of the differences in somatometric data between male Japanese macaques reared in enclosures and cages

\begin{tabular}{|c|c|c|c|c|c|c|c|c|}
\hline \multirow[b]{2}{*}{ Somatometric item } & \multicolumn{8}{|c|}{ Age (year) } \\
\hline & group & 1 & 2 & 3 & 4 & 5 & 6 & 7 \\
\hline \multicolumn{9}{|l|}{ Head } \\
\hline \multirow{2}{*}{ Head Length (1) } & E & $84.6 \pm 2.9$ & $87.7 \pm 4.2$ & $90.4 \pm 1.5$ & $* 96.0 \pm 4.3$ & $97.5 \pm 0.7$ & $100.0 \pm 3.6$ & $100.3 \pm 3.2$ \\
\hline & $\mathrm{C}$ & $81.0 \pm 3.3$ & $83.5 \pm 3.4$ & $88.2 \pm 4.2$ & $91.0 \pm 3.2$ & $94.7 \pm 3.4$ & $97.3 \pm 3.5$ & $98.5 \pm 4.5$ \\
\hline \multirow{2}{*}{ Head Breadth (3) } & E & $68.3 \pm 2.3$ & $70.0 \pm 2.8$ & $73.6 \pm 1.1$ & $76.8 \pm 4.5$ & $77.5 \pm 0.7$ & $80.0 \pm 2.4$ & $82.7 \pm 2.5$ \\
\hline & $\mathrm{C}$ & $64.7 \pm 2.7$ & $68.0 \pm 2.7$ & $71.2 \pm 3.6$ & $74.3 \pm 3.9$ & $77.7 \pm 2.9$ & $79.7 \pm 2.7$ & $80.8 \pm 3.1$ \\
\hline Bizygomatic Breadth (6) & E & $66.4 \pm 2.8$ & $72.3 \pm 3.7$ & $77.4 \pm 1.1$ & $84.0 \pm 3.9$ & $86.5 \pm 2.1$ & $90.8 \pm 2.2$ & $95.3 \pm 3.5$ \\
\hline & $\mathrm{C}$ & $63.4 \pm 3.0$ & $70.7 \pm 2.3$ & $76.7 \pm 2.3$ & $81.7 \pm 2.9$ & $87.8 \pm 2.0$ & $92.8 \pm 2.5$ & $96.0 \pm 1.7$ \\
\hline Head Height (15) & E & $88.3 \pm 4.0$ & $96.4 \pm 2.7$ & $103.8 \pm 2.9$ & $104.3 \pm 19.0$ & $115.0 \pm 4.2$ & $119.5 \pm 5.4$ & $* 125.7 \pm 2.1$ \\
\hline & $\mathrm{C}$ & $87.6 \pm 5.5$ & $92.5 \pm 3.8$ & $100.0 \pm 3.5$ & $106.5 \pm 4.4$ & $112.2 \pm 5.0$ & $117.5 \pm 11.1$ & $118.3 \pm 8.1$ \\
\hline Face & & & & & & & & \\
\hline Facial Height (18) & E & $48.4 \pm 1.7$ & $55.9 \pm 2.4$ & $60.6 \pm 2.1$ & $* * 72.0 \pm 9.9$ & $71.0 \pm 2.8$ & $75.5 \pm 1.3$ & $79.0 \pm 1.7$ \\
\hline & $\mathrm{C}$ & $46.1 \pm 2.0$ & $53.2 \pm 2.2$ & $58.3 \pm 3.7$ & $64.5 \pm 5.2$ & $71.2 \pm 5.1$ & $75.7 \pm 5.6$ & $80.5 \pm 8.0$ \\
\hline Upper Facial Height (20) & E & $29.4 \pm 1.2$ & $34.3 \pm 1.6$ & $39.2 \pm 2.2$ & $43.3 \pm 5.3$ & $47.0 \pm 1.4$ & $51.5 \pm 1.7$ & $57.7 \pm 2.3$ \\
\hline & $\mathrm{C}$ & $28.7 \pm 1.8$ & $33.7 \pm 2.9$ & $36.5 \pm 3.1$ & $41.2 \pm 3.3$ & $46.7 \pm 4.2$ & $52.3 \pm 6.1$ & $55.7 \pm 7.7$ \\
\hline Bigonial Breadth (8) & E & $30.0 \pm 1.4$ & $33.0 \pm 2.1$ & $37.2 \pm 3.2$ & $40.3 \pm 1.7$ & * $39.5 \pm 0.7$ & $* * 42.8 \pm 5.5$ & $47.7 \pm 4.5$ \\
\hline & $\mathrm{C}$ & $30.0 \pm 2.4$ & $31.2 \pm 1.2$ & $35.3 \pm 2.1$ & $39.2 \pm 2.3$ & $43.8 \pm 2.9$ & $48.3 \pm 2.3$ & $46.7 \pm 2.7$ \\
\hline Trunk & & & & & & & & \\
\hline Sitting Height (23) & E & $334.6 \pm 17.1$ & $401.1 \pm 15.5$ & $438.6 \pm 9.8$ & $487.3 \pm 34.8$ & $512.5 \pm 6.4$ & $* 547.3 \pm 15.5$ & $580.3 \pm 25.5$ \\
\hline & $\mathrm{C}$ & $337.1 \pm 23.9$ & $396.2 \pm 28.4$ & $446.5 \pm 27.4$ & $492.7 \pm 30.1$ & $536.5 \pm 34.0$ & $574.2 \pm 29.8$ & $590.5 \pm 30.3$ \\
\hline Tail Length (28(5)) & $\mathrm{E}$ & $64.9 \pm 8.4$ & $75.4 \pm 11.1$ & $82.4 \pm 14.7$ & $87.3 \pm 15.2$ & $94.0 \pm 0.0$ & $105.0 \pm 11.5$ & $99.0 \pm 8.7$ \\
\hline & $\mathrm{C}$ & $69.6 \pm 9.3$ & $78.8 \pm 9.9$ & $89.5 \pm 10.9$ & $96.7 \pm 11.1$ & $104.2 \pm 11.9$ & $107.5 \pm 11.6$ & $106.8 \pm 13.1$ \\
\hline Anterior Trunk Length (27(1a)) & $\mathrm{E}$ & $215.1 \pm 13.0$ & $254.0 \pm 10.7$ & $288.4 \pm 16.0$ & $323.8 \pm 26.4$ & * $343.0 \pm 11.3$ & $360.3 \pm 20.1$ & $398.7 \pm 6.1$ \\
\hline & $\mathrm{C}$ & $218.3 \pm 17.5$ & $267.0 \pm 18.4$ & $302.0 \pm 19.8$ & $336.0 \pm 22.8$ & $370.5 \pm 23.2$ & $381.8 \pm 21.7$ & $402.0 \pm 26.5$ \\
\hline Bi-acromial Breadth (35) & $\mathrm{E}$ & $98.2 \pm 5.6$ & $108.4 \pm 10.4$ & $113.0 \pm 6.5$ & $124.3 \pm 8.4$ & $145.5 \pm 0.7$ & $144.5 \pm 5.4$ & $153.0 \pm 7.5$ \\
\hline & $\mathrm{C}$ & $93.3 \pm 5.8$ & $107.8 \pm 5.1$ & $118.0 \pm 9.2$ & $127.5 \pm 10.7$ & $141.5 \pm 10.6$ & $142.0 \pm 13.4$ & $151.5 \pm 14.4$ \\
\hline Chest Breadth (36) & E & $* 65.3 \pm 6.1$ & $* 76.9 \pm 5.0$ & $85.0 \pm 5.2$ & $90.5 \pm 8.9$ & $94.5 \pm 9.2$ & $100.3 \pm 1.7$ & $106.7 \pm 6.5$ \\
\hline & $\mathrm{C}$ & $56.4 \pm 4.8$ & $68.0 \pm 3.2$ & $78.7 \pm 3.2$ & $85.2 \pm 6.3$ & $93.2 \pm 6.9$ & $101.7 \pm 6.9$ & $101.3 \pm 7.3$ \\
\hline Chest Depth (37) & $\mathrm{E}$ & * $79.9 \pm 5.2$ & $* * 91.9 \pm 7.8$ & $* 100.6 \pm 5.6$ & $* * 113.5 \pm 5.7$ & $109.5 \pm 2.1$ & $120.8 \pm 9.4$ & $125.0 \pm 6.1$ \\
\hline & $\mathrm{C}$ & $69.0 \pm 6.8$ & $77.3 \pm 5.7$ & $90.5 \pm 4.7$ & $101.5 \pm 7.5$ & $115.2 \pm 7.7$ & $121.5 \pm 9.8$ & $121.3 \pm 4.9$ \\
\hline Bi-iliac Breadth (40) & $\mathrm{E}$ & $61.9 \pm 3.2$ & $72.7 \pm 2.1$ & $83.4 \pm 3.3$ & $92.5 \pm 5.8$ & $* * 94.0 \pm 1.4$ & $* * 101.8 \pm 5.6$ & $114.0 \pm 8.0$ \\
\hline & $\mathrm{C}$ & $60.9 \pm 1.7$ & $73.5 \pm 3.1$ & $84.5 \pm 4.0$ & $94.0 \pm 5.2$ & $106.5 \pm 6.5$ & $114.2 \pm 6.9$ & $117.8 \pm 7.1$ \\
\hline Bitrochanteric Breadth (42) & $\mathrm{E}$ & $77.4 \pm 5.5$ & $89.4 \pm 4.6$ & $100.0 \pm 1.2$ & $113.8 \pm 8.2$ & $* 118.5 \pm 2.1$ & $* 128.8 \pm 12.6$ & $139.3 \pm 11.0$ \\
\hline & $\mathrm{C}$ & $72.1 \pm 5.0$ & $85.0 \pm 5.9$ & $99.8 \pm 6.4$ & $113.3 \pm 9.2$ & $129.3 \pm 9.4$ & $138.2 \pm 11.1$ & $142.8 \pm 7.2$ \\
\hline Limbs & & & & & & & & \\
\hline Upper Arm Length (47) & $\mathrm{E}$ & $94.9 \pm 4.6$ & $110.4 \pm 2.1$ & $122.8 \pm 3.8$ & $136.5 \pm 7.9$ & $152.0 \pm 11.3$ & $* 159.3 \pm 2.1$ & $* * 165.0 \pm 3.5$ \\
\hline & $\mathrm{C}$ & $94.0 \pm 6.0$ & $109.8 \pm 7.5$ & $123.5 \pm 7.2$ & $136.7 \pm 7.9$ & $154.2 \pm 11.4$ & $168.2 \pm 10.5$ & $177.7 \pm 9.7$ \\
\hline Forearm Length (48) & $\mathrm{E}$ & $97.2 \pm 7.2$ & $115.6 \pm 4.1$ & $125.8 \pm 3.3$ & $141.0 \pm 11.0$ & $157.0 \pm 4.2$ & $165.5 \pm 5.4$ & $169.7 \pm 5.0$ \\
\hline & $\mathrm{C}$ & $93.7 \pm 6.9$ & $108.7 \pm 7.3$ & $124.5 \pm 8.9$ & $135.7 \pm 7.5$ & $155.0 \pm 7.5$ & $165.5 \pm 7.7$ & $167.7 \pm 6.7$ \\
\hline Hand Length (49) & $\mathrm{E}$ & $* 82.7 \pm 4.7$ & $* 92.6 \pm 4.8$ & $101.2 \pm 3.9$ & $* 111.0 \pm 5.3$ & $* 119.5 \pm 2.1$ & $123.5 \pm 1.7$ & $* * 128.3 \pm 5.5$ \\
\hline & $\mathrm{C}$ & $75.9 \pm 3.9$ & $84.7 \pm 5.3$ & $95.3 \pm 4.0$ & $104.3 \pm 5.6$ & $112.3 \pm 6.9$ & $118.3 \pm 4.4$ & $118.3 \pm 4.3$ \\
\hline Hand Breadth (52) & $\mathrm{E}$ & $* 30.9 \pm 1.5$ & $* 33.4 \pm 2.4$ & $36.6 \pm 3.1$ & $39.0 \pm 2.6$ & $41.5 \pm 0.7$ & $* * 44.8 \pm 0.5$ & * $44.3 \pm 2.1$ \\
\hline & $\mathrm{C}$ & $28.0 \pm 1.3$ & $30.8 \pm 2.1$ & $34.3 \pm 1.6$ & $36.5 \pm 2.8$ & $40.0 \pm 1.8$ & $41.2 \pm 1.7$ & $41.3 \pm 1.0$ \\
\hline 1st Digit Length (51a) & $\mathrm{E}$ & $16.4 \pm 0.5$ & $17.9 \pm 1.8$ & $20.0 \pm 2.1$ & $21.8 \pm 2.2$ & $23.5 \pm 0.7$ & $24.5 \pm 0.6$ & * $26.3 \pm 2.1$ \\
\hline & $\mathrm{C}$ & $14.9 \pm 0.9$ & $16.3 \pm 1.4$ & $18.2 \pm 1.7$ & $20.0 \pm 1.3$ & $21.5 \pm 1.8$ & $22.8 \pm 1.7$ & $23.8 \pm 1.9$ \\
\hline 3rd Digit Length (51) & $\mathrm{E}$ & $38.9 \pm 2.0$ & $43.1 \pm 3.0$ & $48.6 \pm 3.2$ & $51.8 \pm 2.9$ & $* 58.0 \pm 1.4$ & $58.3 \pm 2.4$ & $59.7 \pm 3.2$ \\
\hline & $\mathrm{C}$ & $36.9 \pm 2.2$ & $40.3 \pm 3.4$ & $45.0 \pm 2.0$ & $48.7 \pm 3.3$ & $53.0 \pm 3.8$ & $55.5 \pm 2.7$ & $58.0 \pm 5.6$ \\
\hline Thigh Length (55(1)) & $\mathrm{E}$ & $115.2 \pm 7.3$ & $138.3 \pm 5.9$ & $154.4 \pm 5.6$ & $174.0 \pm 10.8$ & $194.5 \pm 6.4$ & $205.0 \pm 7.5$ & $208.3 \pm 6.5$ \\
\hline & $\mathrm{C}$ & $113.3 \pm 8.3$ & $137.3 \pm 9.4$ & $157.7 \pm 9.8$ & $175.3 \pm 9.8$ & $198.3 \pm 14.2$ & $207.8 \pm 6.9$ & $216.3 \pm 5.9$ \\
\hline Leg Length (56a) & $\mathrm{E}$ & $97.0 \pm 7.5$ & $113.6 \pm 5.6$ & $125.4 \pm 6.0$ & $141.0 \pm 15.5$ & $156.0 \pm 8.5$ & $166.5 \pm 5.3$ & $173.7 \pm 5.0$ \\
\hline & $\mathrm{C}$ & $94.6 \pm 6.7$ & $111.0 \pm 6.4$ & $127.5 \pm 8.9$ & $142.5 \pm 9.5$ & $159.8 \pm 10.5$ & $169.2 \pm 8.1$ & $173.0 \pm 8.0$ \\
\hline Foot Length (58) & $\mathrm{E}$ & $115.4 \pm 6.7$ & $* 130.9 \pm 6.7$ & $142.6 \pm 4.0$ & $155.8 \pm 7.8$ & $168.0 \pm 7.1$ & $* 176.8 \pm 3.4$ & $* * 181.7 \pm 6.8$ \\
\hline & $\mathrm{C}$ & $106.4 \pm 7.3$ & $119.7 \pm 9.2$ & $133.8 \pm 7.7$ & $145.8 \pm 10.0$ & $158.8 \pm 8.9$ & $163.5 \pm 7.6$ & $166.7 \pm 7.4$ \\
\hline Foot Breadth & $\mathrm{E}$ & $* 30.1 \pm 2.0$ & $* \quad 33.0 \pm 1.9$ & $36.0 \pm 1.4$ & $* 40.0 \pm 2.0$ & $42.0 \pm 0.0$ & $42.8 \pm 1.5$ & $* * \quad 46.3 \pm 2.9$ \\
\hline & $\mathrm{C}$ & $27.1 \pm 1.9$ & $30.3 \pm 1.4$ & $34.5 \pm 1.6$ & $36.8 \pm 1.8$ & $40.2 \pm 2.1$ & $41.2 \pm 2.2$ & $41.2 \pm 1.7$ \\
\hline 1st Toe Length & $\mathrm{E}$ & $20.4 \pm 1.7$ & $22.9 \pm 2.4$ & $26.0 \pm 1.0$ & $28.5 \pm 2.1$ & $29.0 \pm 2.8$ & $30.3 \pm 1.3$ & * $33.0 \pm 1.7$ \\
\hline & $\mathrm{C}$ & $19.6 \pm 1.3$ & $21.2 \pm 2.6$ & $24.0 \pm 1.4$ & $26.3 \pm 2.3$ & $28.0 \pm 1.7$ & $30.2 \pm 1.2$ & $30.8 \pm 1.2$ \\
\hline 3rd Toe Length & $\mathrm{E}$ & $41.3 \pm 2.1$ & $46.4 \pm 3.8$ & $51.2 \pm 2.5$ & $55.8 \pm 2.2$ & $* 63.0 \pm 0.0$ & $63.3 \pm 1.7$ & * $64.7 \pm 2.5$ \\
\hline & $\mathrm{C}$ & $38.9 \pm 2.8$ & $43.2 \pm 3.5$ & $48.7 \pm 3.1$ & $52.8 \pm 3.9$ & $57.5 \pm 3.9$ & $59.0 \pm 3.7$ & $59.7 \pm 3.4$ \\
\hline Girth & & & & & & & & \\
\hline Chest Girth (61) & $\mathrm{E}$ & $* * 259.3 \pm 17.5$ & $* * 296.0 \pm 14.3$ & $* 321.0 \pm 6.9$ & $* * 359.0 \pm 22.6$ & $362.5 \pm 17.7$ & $398.3 \pm 13.5$ & $415.3 \pm 21.0$ \\
\hline & $\mathrm{C}$ & $228.4 \pm 14.7$ & $263.5 \pm 14.3$ & $295.8 \pm 13.9$ & $331.7 \pm 23.4$ & $364.2 \pm 15.4$ & $391.8 \pm 20.6$ & $405.7 \pm 9.4$ \\
\hline Abdominal Girth (62(1)) & $\mathrm{E}$ & * $201.8 \pm 19.3$ & $* * 252.4 \pm 19.9$ & $256.6 \pm 21.9$ & $280.3 \pm 32.6$ & $273.0 \pm 5.7$ & $290.8 \pm 18.5$ & $308.7 \pm 19.9$ \\
\hline & $\mathrm{C}$ & $173.6 \pm 14.2$ & $203.0 \pm 11.2$ & $231.7 \pm 22.7$ & $252.2 \pm 17.9$ & $278.8 \pm 13.8$ & $296.7 \pm 14.8$ & $297.5 \pm 14.7$ \\
\hline Max. Upper Arm Girth (65) & $\mathrm{E}$ & $92.2 \pm 31.6$ & $116.3 \pm 12.7$ & $131.2 \pm 5.3$ & $148.8 \pm 16.3$ & $149.0 \pm 8.5$ & $164.8 \pm 8.5$ & * $178.0 \pm 14.4$ \\
\hline & $\mathrm{C}$ & $89.0 \pm 6.1$ & $104.5 \pm 4.6$ & $117.8 \pm 6.3$ & $133.3 \pm 12.3$ & $147.5 \pm 10.3$ & $154.8 \pm 15.9$ & $157.8 \pm 10.8$ \\
\hline Max. Forearm Girth (66) & $\mathrm{E}$ & $* * 95.2 \pm 5.2$ & $* 108.6 \pm 6.0$ & $* 116.4 \pm 7.7$ & $* * 127.8 \pm 11.2$ & $134.0 \pm 7.1$ & $* 143.5 \pm 3.1$ & $* * 148.0 \pm 5.2$ \\
\hline & $\mathrm{C}$ & $80.4 \pm 6.7$ & $95.7 \pm 3.4$ & $105.2 \pm 6.9$ & $112.5 \pm 10.3$ & $127.2 \pm 7.2$ & $132.3 \pm 10.2$ & $134.0 \pm 14.6$ \\
\hline Min. Forearm Girth (67) & E & $* * 69.6 \pm 4.2$ & $* * 74.3 \pm 4.2$ & $* * 80.6 \pm 4.5$ & ** $86.0 \pm 3.4$ & $* * 91.0 \pm 2.8$ & ** $94.8 \pm 2.9$ & * $93.7 \pm 4.5$ \\
\hline & $\mathrm{C}$ & $59.6 \pm 3.6$ & $64.0 \pm 3.3$ & $69.7 \pm 4.5$ & $75.7 \pm 5.3$ & $82.5 \pm 4.0$ & $85.5 \pm 5.0$ & $86.5 \pm 3.0$ \\
\hline Max. Thigh Girth (68) & $\mathrm{E}$ & $137.3 \pm 8.7$ & $163.7 \pm 12.5$ & *185.4 8.6 & *208.8 \pm 19.6 & $231.0 \pm 15.6$ & $234.3 \pm 7.0$ & $246.7 \pm 6.7$ \\
\hline & $\mathrm{C}$ & $120.7 \pm 7.8$ & $148.5 \pm 6.8$ & $165.8 \pm 9.2$ & $188.3 \pm 14.8$ & $213.0 \pm 14.7$ & $221.8 \pm 27.3$ & $230.8 \pm 19.7$ \\
\hline Max. Leg Girth (69) & $\mathrm{E}$ & $* * 104.7 \pm 5.9$ & $* * 118.0 \pm 8.2$ & $* 122.8 \pm 4.7$ & $* 134.8 \pm 6.7$ & $139.0 \pm 1.4$ & $149.3 \pm 2.2$ & $* * 157.3 \pm 10.7$ \\
\hline & $\mathrm{C}$ & $81.3 \pm 6.4$ & $99.0 \pm 7.2$ & $109.8 \pm 6.2$ & $121.5 \pm 10.6$ & $137.2 \pm 7.4$ & $140.2 \pm 14.7$ & $141.8 \pm 11.8$ \\
\hline Min. Leg Girth (70) & $\mathrm{E}$ & ** $73.2 \pm 4.3$ & $* * 80.1 \pm 7.2$ & $* * 86.8 \pm 4.4$ & ** $93.8 \pm 8.9$ & $91.0 \pm 2.8$ & $* 96.8 \pm 1.9$ & $* * 103.7 \pm 4.0$ \\
\hline & $\mathrm{C}$ & $61.4 \pm 4.6$ & $67.5 \pm 3.7$ & $73.5 \pm 5.6$ & $79.7 \pm 6.3$ & $85.7 \pm 4.7$ & $88.3 \pm 6.9$ & $90.8 \pm 6.6$ \\
\hline Body Weight (71) & $\mathrm{E}$ & $2.3 \pm 0.3$ & $3.5 \pm 0.4$ & $4.3 \pm 0.2$ & $6.0 \pm 1.2$ & $6.9 \pm 0.2$ & $8.2 \pm 0.6$ & * $\quad 9.6 \pm 1.0$ \\
\hline & $\mathrm{C}$ & $1.8 \pm 0.3$ & $2.9 \pm 0.3$ & $4.1 \pm 0.6$ & $5.4 \pm 0.8$ & $7.0 \pm 0.7$ & $8.2 \pm 0.9$ & $8.7 \pm 0.8$ \\
\hline
\end{tabular}

Somatometric items were chosen after Martin and Saller [7]. Numbers in parentheses correspond to those used by those authors. Three additional limbs measurements, foot breadth, 1st toe length and 3rd toe length, were chosen from Hamada [1] and Iwamoto [6]. The growth pattern of each somatometric item is specified based on differences in somatometric data between the Enclosure and Caged group macaques. Detailed descriptions of these growth categories are given in the text. Differences between the found are highlighted. $*: \mathrm{p}<0.05, * * \mathrm{p}<0.01$, E: Enclosure group, C: Caged group. 
Table 3. Summary of the differences in somatometric data between female Japanese macaques reared in enclosures and cages

\begin{tabular}{|c|c|c|c|c|c|c|c|c|}
\hline \multirow[b]{2}{*}{ Somatometric item } & \multicolumn{8}{|c|}{ Age (year) } \\
\hline & group & 1 & 2 & 3 & 4 & 5 & 6 & 7 \\
\hline \multirow{2}{*}{$\begin{array}{l}\text { Head } \\
\text { Head Length (1) }\end{array}$} & $\mathrm{E}$ & $* 82.7 \pm 3.0$ & $86.0 \pm 2.3$ & $88.7 \pm 2.5$ & * $92.6 \pm 4.5$ & $* 94.9 \pm 3.5$ & $* * 96.8+22$ & $* 97.3 \pm 2.8$ \\
\hline & $\mathrm{C}$ & $78.0 \pm 2.4$ & $81.3 \pm 3.0$ & $84.2 \pm 4.0$ & $86.8 \pm 3.8$ & $89.7 \pm 5.2$ & $90.5 \pm 3.9$ & $91.5 \pm 5.1$ \\
\hline \multirow{2}{*}{ Head Breadth (3) } & $\mathrm{E}$ & $* 65.6 \pm 2.6$ & $69.0 \pm 2.1$ & * $72.6 \pm 2.5$ & $* * 74.7 \pm 1.7$ & * $76.3 \pm 1.7$ & $* * 77.8 \pm 2.2$ & $76.3 \pm 2.1$ \\
\hline & $\mathrm{C}$ & $62.0 \pm 2.2$ & $65.7 \pm 2.4$ & $68.0 \pm 2.1$ & $69.3 \pm 2.1$ & $72.2 \pm 4.7$ & $72.0 \pm 2.9$ & $73.0 \pm 2.7$ \\
\hline \multirow[t]{2}{*}{ Bizygomatic Breadth (6) } & $\mathrm{E}$ & $63.6 \pm 3.0$ & $70.1 \pm 1.8$ & $* 75.9 \pm 2.3$ & $* * 82.1 \pm 3.1$ & $* * 84.3 \pm 2.6$ & $* * 86.0 \pm 2.3$ & $86.0 \pm 2.2$ \\
\hline & $\mathrm{C}$ & $60.4 \pm 3.2$ & $67.3 \pm 3.4$ & $71.8 \pm 3.2$ & $75.7 \pm 4.0$ & $78.7 \pm 5.0$ & $80.5 \pm 4.6$ & $83.8 \pm 4.6$ \\
\hline \multirow[t]{2}{*}{ Head Height (15) } & E & $85.9 \pm 3.2$ & $93.4 \pm 5.0$ & $100.7 \pm 4.2$ & $* * 109.6 \pm 4.9$ & $* * 110.6 \pm 4.6$ & $* 112.5 \pm 3.9$ & $* * 114.5 \pm 3.0$ \\
\hline & $\mathrm{C}$ & $81.9 \pm 4.1$ & $87.2 \pm 4.0$ & $93.3 \pm 4.9$ & $95.7 \pm 7.5$ & $99.7 \pm 3.2$ & $103.0 \pm 4.4$ & $101.0 \pm 5.0$ \\
\hline Face & & & & & & & & \\
\hline Facial Height (18) & $\mathrm{E}$ & $46.2 \pm 2.7$ & $53.6 \pm 3.5$ & $* 59.3 \pm 2.9$ & $* * 66.3 \pm 2.4$ & $* 66.9 \pm 3.1$ & $* * 72.8 \pm 1.0$ & $* * 72.8 \pm 2.4$ \\
\hline & $\mathrm{C}$ & $44.9 \pm 2.4$ & $50.0 \pm 2.5$ & $53.7 \pm 1.0$ & $58.3 \pm 3.2$ & $60.8 \pm 2.6$ & $62.7 \pm 2.7$ & $63.5 \pm 3.3$ \\
\hline Upper Facial Height (20) & $\mathrm{E}$ & $28.3 \pm 1.6$ & $34.4 \pm 2.0$ & $* 38.4 \pm 2.6$ & $* 43.3 \pm 1.4$ & ** $45.6 \pm 3.3$ & $* * 50.5 \pm 2.4$ & $* * 51.7 \pm 2.1$ \\
\hline & $\mathrm{C}$ & $28.1 \pm 0.9$ & $32.0 \pm 2.4$ & $34.0 \pm 3.5$ & $38.5 \pm 3.3$ & $39.8 \pm 4.2$ & $41.8 \pm 3.4$ & $44.3 \pm 4.6$ \\
\hline Bigonial Breadth (8) & $\mathrm{E}$ & $29.2 \pm 1.9$ & $33.8 \pm 2.3$ & $36.9 \pm 3.0$ & $39.1 \pm 2.0$ & $41.1 \pm 3.0$ & $39.3 \pm 1.7$ & $40.8 \pm 3.8$ \\
\hline & $\mathrm{C}$ & $29.3 \pm 2.2$ & $32.0 \pm 1.8$ & $34.8 \pm 5.2$ & $37.7 \pm 4.8$ & $39.8 \pm 2.0$ & $41.8 \pm 4.0$ & $43.0 \pm 5.5$ \\
\hline Trunk & & & & & & & & \\
\hline Sitting Height (23) & $\mathrm{E}$ & $335.2 \pm 18.6$ & $388.0 \pm 14.1$ & $446.4 \pm 13.7$ & $480.4 \pm 22.6$ & $508.4 \pm 18.4$ & $* 527.0 \pm 15.8$ & $518.5 \pm 18.3$ \\
\hline & $\mathrm{C}$ & $325.0 \pm 16.1$ & $379.2 \pm 21.5$ & $419.7 \pm 27.0$ & $455.3 \pm 27.3$ & $486.7 \pm 17.8$ & $487.7 \pm 22.9$ & $520.8 \pm 16.5$ \\
\hline Tail Length (28(5)) & $\mathrm{E}$ & $66.3 \pm 5.3$ & $78.5 \pm 7.0$ & $88.4 \pm 10.3$ & $87.1 \pm 5.6$ & $90.3 \pm 11.7$ & $97.3 \pm 1.0$ & $84.5 \pm 12.0$ \\
\hline & $\mathrm{C}$ & $68.6 \pm 5.5$ & $80.8 \pm 8.8$ & $86.2 \pm 11.7$ & $94.2 \pm 13.6$ & $94.2 \pm 8.9$ & $96.0 \pm 11.8$ & $95.0 \pm 14.0$ \\
\hline Anterior Trunk Length (27(1a)) & $\mathrm{E}$ & $215.6 \pm 12.1$ & $258.1 \pm 13.1$ & $297.6 \pm 13.8$ & $322.3 \pm 22.5$ & $340.0 \pm 21.4$ & $358.8 \pm 14.7$ & $346.3 \pm 16.9$ \\
\hline & $\mathrm{C}$ & $211.7 \pm 10.9$ & $252.2 \pm 13.7$ & $282.3 \pm 19.1$ & $306.8 \pm 20.6$ & $328.8 \pm 16.1$ & $338.8 \pm 18.9$ & $357.8 \pm 15.1$ \\
\hline Bi-acromial Breadth (35) & $\mathrm{E}$ & $89.5 \pm 9.8$ & $103.8 \pm 6.2$ & $110.6 \pm 6.9$ & $125.3 \pm 11.2$ & $131.0 \pm 6.8$ & $134.0 \pm 12.2$ & $134.0 \pm 11.5$ \\
\hline & $\mathrm{C}$ & $87.4 \pm 9.5$ & $100.8 \pm 8.8$ & $114.0 \pm 11.5$ & $117.8 \pm 10.6$ & $122.3 \pm 11.0$ & $128.0 \pm 10.6$ & $131.0 \pm 11.9$ \\
\hline Chest Breadth (36) & $\mathrm{E}$ & $* 63.9 \pm 3.8$ & $72.3 \pm 6.0$ & $78.7 \pm 4.2$ & $* 90.6 \pm 7.3$ & $* 94.0 \pm 6.2$ & $* 100.5 \pm 2.6$ & $98.8 \pm 6.9$ \\
\hline & $\mathrm{C}$ & $54.4 \pm 5.5$ & $67.2 \pm 5.4$ & $72.0 \pm 7.1$ & $80.7 \pm 7.1$ & $84.7 \pm 6.1$ & $90.0 \pm 7.7$ & $94.5 \pm 7.0$ \\
\hline Chest Depth (37) & $\mathrm{E}$ & $76.2 \pm 6.2$ & * $89.3 \pm 7.0$ & $93.4 \pm 6.1$ & $103.9 \pm 6.9$ & $107.0 \pm 4.9$ & $* * 117.5 \pm 4.5$ & * $116.3 \pm 8.4$ \\
\hline & $\mathrm{C}$ & $68.4 \pm 8.1$ & $80.5 \pm 6.3$ & $85.8 \pm 6.9$ & $96.5 \pm 7.1$ & $100.3 \pm 4.0$ & $105.8 \pm 3.6$ & $107.5 \pm 4.5$ \\
\hline Bi-iliac Breadth (40) & $\mathrm{E}$ & $59.8 \pm 4.1$ & $70.0 \pm 3.4$ & $79.9 \pm 2.0$ & $88.6 \pm 4.7$ & $95.7 \pm 6.0$ & $99.8 \pm 5.6$ & $* * 94.8 \pm 6.5$ \\
\hline & $\mathrm{C}$ & $59.7 \pm 2.5$ & $70.3 \pm 4.3$ & $80.3 \pm 4.4$ & $89.3 \pm 5.0$ & $96.3 \pm 5.5$ & $100.5 \pm 4.0$ & $103.5 \pm 3.0$ \\
\hline Bitrochanteric Breadth (42) & $\mathrm{E}$ & $73.4 \pm 4.6$ & $87.0 \pm 4.3$ & $97.0 \pm 4.0$ & $109.4 \pm 8.8$ & $119.0 \pm 6.8$ & $124.5 \pm 7.3$ & $123.2 \pm 6.1$ \\
\hline & $\mathrm{C}$ & $69.3 \pm 3.9$ & $82.2 \pm 6.9$ & $92.8 \pm 10.2$ & $103.7 \pm 9.0$ & $111.2 \pm 8.6$ & $119.0 \pm 7.8$ & $122.0 \pm 11.2$ \\
\hline Limbs & & & & & & & & \\
\hline Upper Arm Length (47) & $\mathrm{E}$ & $92.7 \pm 6.3$ & $108.9 \pm 4.5$ & $122.4 \pm 6.8$ & $137.7 \pm 6.3$ & $145.9 \pm 5.8$ & $151.3 \pm 4.2$ & $149.5 \pm 7.1$ \\
\hline & $\mathrm{C}$ & $91.1 \pm 5.9$ & $106.7 \pm 4.3$ & $119.2 \pm 5.2$ & $131.3 \pm 6.1$ & $139.0 \pm 6.8$ & $143.2 \pm 8.1$ & $148.8 \pm 5.5$ \\
\hline Forearm Length (48) & $\mathrm{E}$ & $95.7 \pm 5.1$ & $113.4 \pm 4.8$ & $125.9 \pm 6.1$ & $* 142.1 \pm 4.0$ & $150.0 \pm 6.4$ & $* * 155.5 \pm 3.1$ & $156.8 \pm 7.8$ \\
\hline & $\mathrm{C}$ & $92.9 \pm 5.0$ & $108.8 \pm 5.6$ & $122.0 \pm 11.0$ & $132.2 \pm 6.5$ & $141.5 \pm 6.2$ & $144.2 \pm 5.2$ & $149.3 \pm 4.4$ \\
\hline Hand Length (49) & $\mathrm{E}$ & $79.7 \pm 2.5$ & $* 90.5 \pm 3.6$ & $* 99.9 \pm 4.9$ & $* 110.9 \pm 5.1$ & $* * 111.4 \pm 3.2$ & $* * 113.0 \pm 3.6$ & $* * 113.2 \pm 5.1$ \\
\hline & $\mathrm{C}$ & $74.3 \pm 3.6$ & $83.8 \pm 5.9$ & $92.2 \pm 7.5$ & $97.8 \pm 7.0$ & $102.2 \pm 5.2$ & $104.5 \pm 5.0$ & $104.3 \pm 6.4$ \\
\hline Hand Breadth (52) & $\mathrm{E}$ & * $29.8 \pm 1.7$ & $* * 32.6 \pm 1.0$ & $* * 35.1 \pm 1.7$ & $* * 38.3 \pm 1.5$ & $\begin{array}{lll}* & 39.4 \pm 1.7\end{array}$ & $* * 40.0 \pm 2.4$ & $* * \quad 40.5 \pm 1.6$ \\
\hline & $\mathrm{C}$ & $26.7 \pm 1.0$ & $29.2 \pm 1.9$ & $31.5 \pm 2.7$ & $34.7 \pm 2.3$ & $35.8 \pm 2.7$ & $35.8 \pm 2.2$ & $37.0 \pm 2.6$ \\
\hline 1st Digit Length (51a) & $\mathrm{E}$ & $15.7 \pm 1.2$ & $17.9 \pm 1.4$ & $19.1 \pm 1.1$ & $* * \quad 21.4 \pm 1.3$ & $21.6 \pm 1.3$ & $* * 23.0 \pm 1.4$ & ** $\quad 22.8 \pm 1.2$ \\
\hline & $\mathrm{C}$ & $15.4 \pm 1.8$ & $16.3 \pm 1.6$ & $18.0 \pm 1.7$ & $18.0 \pm 1.4$ & $19.8 \pm 1.6$ & $20.0 \pm 1.5$ & $20.3 \pm 1.5$ \\
\hline 3rd Digit Length (51) & $\mathrm{E}$ & $38.1 \pm 1.6$ & $43.0 \pm 1.2$ & $47.1 \pm 2.4$ & $* 52.3 \pm 2.1$ & $52.7 \pm 2.4$ & $53.3 \pm 2.2$ & $53.5 \pm 3.1$ \\
\hline & $\mathrm{C}$ & $36.0 \pm 3.6$ & $40.5 \pm 3.4$ & $43.5 \pm 4.1$ & $47.0 \pm 5.1$ & $49.0 \pm 4.1$ & $49.8 \pm 4.2$ & $50.8 \pm 4.1$ \\
\hline Thigh Length (55(1)) & $\mathrm{E}$ & $112.5 \pm 6.2$ & $135.9 \pm 6.2$ & $155.0 \pm 7.9$ & * $174.3 \pm 8.2$ & $180.9 \pm 6.0$ & $183.0 \pm 7.4$ & $185.0 \pm 10.8$ \\
\hline & $\mathrm{C}$ & $110.7 \pm 5.5$ & $132.7 \pm 7.6$ & $148.3 \pm 9.4$ & $163.0 \pm 10.2$ & $173.8 \pm 9.8$ & $179.2 \pm 9.1$ & $182.8 \pm 6.4$ \\
\hline Leg Length (56a) & $\mathrm{E}$ & $94.0 \pm 5.7$ & $113.4 \pm 4.7$ & $127.9 \pm 5.6$ & $* 147.0 \pm 9.3$ & $150.4 \pm 3.2$ & $154.3 \pm 3.6$ & $152.8 \pm 8.3$ \\
\hline & $\mathrm{C}$ & $93.0 \pm 4.6$ & $108.5 \pm 7.7$ & $122.3 \pm 9.4$ & $135.0 \pm 10.0$ & $143.8 \pm 8.7$ & $146.5 \pm 7.4$ & $149.8 \pm 7.9$ \\
\hline Foot Length (58) & $\mathrm{E}$ & $111.4 \pm 6.3$ & * $129.3 \pm 5.6$ & $* 141.3 \pm 5.2$ & $* * 153.6 \pm 5.7$ & * $156.1 \pm 5.9$ & $* 159.3 \pm 6.7$ & $* 161.0 \pm 7.3$ \\
\hline & $\mathrm{C}$ & $104.3 \pm 6.6$ & $118.2 \pm 9.2$ & $129.7 \pm 11.9$ & $137.5 \pm 13.8$ & $144.2 \pm 9.1$ & $147.0 \pm 10.0$ & $148.3 \pm 11.0$ \\
\hline Foot Breadth & $\mathrm{E}$ & * $29.1 \pm 1.7$ & $* 32.6 \pm 1.6$ & $* * 35.4 \pm 1.8$ & $* * \quad 38.7 \pm 2.2$ & $* * \quad 39.7 \pm 1.3$ & $* * \quad 40.5 \pm 2.5$ & $* * 40.8 \pm 1.6$ \\
\hline & $\mathrm{C}$ & $25.9 \pm 0.9$ & $29.7 \pm 1.9$ & $31.7 \pm 2.5$ & $34.5 \pm 2.4$ & $36.0 \pm 2.9$ & $36.5 \pm 2.8$ & $37.0 \pm 3.4$ \\
\hline 1st Toe Length & $\mathrm{E}$ & $20.6 \pm 1.6$ & $22.6 \pm 1.3$ & $25.6 \pm 1.0$ & $27.1 \pm 1.9$ & $27.7 \pm 2.4$ & $28.0 \pm 1.2$ & $27.8 \pm 0.8$ \\
\hline & $\mathrm{C}$ & $19.0 \pm 1.5$ & $21.7 \pm 1.5$ & $23.7 \pm 1.9$ & $25.0 \pm 2.3$ & $26.2 \pm 1.3$ & $27.5 \pm 1.0$ & $27.3 \pm 1.0$ \\
\hline 3rd Toe Length & $\mathrm{E}$ & $40.6 \pm 2.3$ & $46.2 \pm 2.6$ & $51.6 \pm 1.4$ & $* * 56.3 \pm 2.3$ & $56.9 \pm 2.5$ & $57.5 \pm 2.4$ & $58.5 \pm 3.4$ \\
\hline & $\mathrm{C}$ & $39.0 \pm 2.7$ & $43.5 \pm 3.6$ & $47.8 \pm 4.9$ & $50.2 \pm 5.7$ & $52.7 \pm 4.4$ & $53.5 \pm 4.4$ & $54.3 \pm 4.6$ \\
\hline Girth & & & & & & & & \\
\hline Chest Girth (61) & $\mathrm{E}$ & $* * 250.1 \pm 15.3$ & $* * 284.0 \pm 11.3$ & $* * 315.4 \pm 12.2$ & $* * 342.3 \pm 16.1$ & $* * 358.4 \pm 17.0$ & $* * 383.5 \pm 15.2$ & $* 381.5 \pm 24.0$ \\
\hline & $\mathrm{C}$ & $216.9 \pm 10.8$ & $252.5 \pm 12.0$ & $273.8 \pm 15.4$ & $307.3 \pm 16.9$ & $324.0 \pm 11.2$ & $342.3 \pm 13.4$ & $357.8 \pm 14.3$ \\
\hline Abdominal Girth (62(1)) & $\mathrm{E}$ & $* 205.3 \pm 18.4$ & $* 233.0 \pm 21.9$ & $* 244.0 \pm 24.0$ & $262.1 \pm 23.8$ & $* 284.9 \pm 23.3$ & $291.3 \pm 24.4$ & $304.6 \pm 34.2$ \\
\hline & $\mathrm{C}$ & $170.4 \pm 18.6$ & $198.3 \pm 19.7$ & $215.4 \pm 17.8$ & $239.3 \pm 19.9$ & $255.3 \pm 20.6$ & $273.7 \pm 17.4$ & $280.5 \pm 18.0$ \\
\hline Max. Upper Arm Girth (65) & $\mathrm{E}$ & $101.8 \pm 10.5$ & $114.6 \pm 9.8$ & $127.9 \pm 8.9$ & $* 142.7 \pm 10.5$ & $147.0 \pm 3.8$ & $151.3 \pm 18.9$ & $144.7 \pm 9.6$ \\
\hline & $\mathrm{C}$ & $85.1 \pm 5.9$ & $98.7 \pm 8.0$ & $112.5 \pm 8.9$ & $122.7 \pm 8.5$ & $130.3 \pm 12.4$ & $140.2 \pm 11.5$ & $141.3 \pm 13.1$ \\
\hline Max. Forearm Girth (66) & $\mathrm{E}$ & * $89.4 \pm 7.8$ & $* * 100.5 \pm 3.9$ & $* 114.1 \pm 5.0$ & $* * 123.7 \pm 6.6$ & $* * 128.4 \pm 7.0$ & $* * 133.0 \pm 7.8$ & $* * 130.5 \pm 3.8$ \\
\hline & $\mathrm{C}$ & $77.9 \pm 7.4$ & $86.3 \pm 8.0$ & $102.0 \pm 9.2$ & $108.5 \pm 6.6$ & $112.7 \pm 9.4$ & $118.0 \pm 8.5$ & $126.8 \pm 7.6$ \\
\hline Min. Forearm Girth (67) & $\mathrm{E}$ & $* * \quad 67.1 \pm 4.9$ & $* * \quad 71.9 \pm 4.4$ & ** $77.4 \pm 3.1$ & ** $\quad 82.1 \pm 5.2$ & ** $\quad 86.1 \pm 3.3$ & $* * \quad 92.5 \pm 3.9$ & $85.5 \pm 5.9$ \\
\hline & $\mathrm{C}$ & $53.9 \pm 3.6$ & $59.2 \pm 5.7$ & $64.7 \pm 5.5$ & $69.3 \pm 3.8$ & $72.0 \pm 5.1$ & $74.3 \pm 5.2$ & $76.0 \pm 4.2$ \\
\hline Max. Thigh Girth (68) & $\mathrm{E}$ & $135.3 \pm 14.3$ & * $162.1 \pm 12.1$ & $* * 184.6 \pm 7.9$ & * $201.1 \pm 16.1$ & $* * 222.9 \pm 9.0$ & $* * 233.8 \pm 17.0$ & $220.3 \pm 18.5$ \\
\hline & $\mathrm{C}$ & $121.3 \pm 8.6$ & $140.7 \pm 12.3$ & $156.5 \pm 16.7$ & $178.8 \pm 13.9$ & $191.3 \pm 16.7$ & $204.8 \pm 18.4$ & $209.8 \pm 19.0$ \\
\hline Max. Leg Girth (69) & $\mathrm{E}$ & $* * 100.8 \pm 8.6$ & * $111.5 \pm 7.2$ & $* 120.9 \pm 6.8$ & ** $\quad 136.0 \pm 9.9$ & $* * 138.4 \pm 10.9$ & $* 143.0 \pm 8.8$ & $145.8 \pm 9.1$ \\
\hline & $\mathrm{C}$ & $77.1 \pm 7.2$ & $99.2 \pm 11.9$ & $105.5 \pm 8.0$ & $111.0 \pm 9.9$ & $121.2 \pm 7.5$ & $130.2 \pm 13.2$ & $140.3 \pm 11.0$ \\
\hline Min. Leg Girth (70) & $\mathrm{E}$ & $\begin{array}{ll}* * & 69.3 \pm 4.8\end{array}$ & $\begin{array}{ll}* * & 78.1 \pm 3.6\end{array}$ & $* * \quad 82.4 \pm 3.9$ & ** $\quad 91.4 \pm 5.3$ & $\begin{array}{ll}* & 89.9 \pm 3.0\end{array}$ & $* * 92.3 \pm 3.5$ & $* * 92.8 \pm 5.5$ \\
\hline & $\mathrm{C}$ & $56.1 \pm 3.0$ & $61.7 \pm 5.4$ & $68.0 \pm 6.3$ & $69.8 \pm 5.5$ & $74.3 \pm 5.4$ & $78.5 \pm 5.1$ & $82.5 \pm 5.4$ \\
\hline Body Weight (71) & $\mathrm{E}$ & $2.1 \pm 0.4$ & $3.0 \pm 0.2$ & $4.3 \pm 0.2$ & $5.6 \pm 1.0$ & $\begin{array}{ll}* * & 6.3 \pm 0.6\end{array}$ & $* * \quad 7.5 \pm 0.6$ & $7.0 \pm 1.0$ \\
\hline & $\mathrm{C}$ & $1.6 \pm 0.2$ & $2.5 \pm 0.4$ & $3.5 \pm 0.7$ & $4.3 \pm 0.7$ & $5.0 \pm 0.8$ & $5.9 \pm 0.8$ & $6.4 \pm 0.8$ \\
\hline
\end{tabular}


significantly larger than that of the Enclosure group. In females, all limb measurements, except upper arm length and 1 st toe length, of the Caged group were significantly smaller than those of the Enclosure group at certain growth stages. This tendency was remarkable in hand and foot, length and breadth in females as well as in males.

Girth: We found the largest number of age-related significant differences between the Enclosure and Caged groups in girth measurements. More significant differences were evident between females than males. For all measurements exhibiting significant differences, the average values of Caged group macaques were smaller than those of the Enclosure group in both sexes. In particular, in maximal and minimal girth of the forearm and maximal and minimal girth of the leg, the Caged group values were smaller than those of the Enclosure group at most measurement points over the 7 years in both sexes. In females, chest girth of the Caged group was significantly smaller than that of the Enclosure group at every year.

Body weight: Body weight of the Caged group was significantly smaller than that of the Enclosure group at 7 years of age in males, and from 4 to 6 years in females.

\section{DISCUSSION}

Differences in growth between Enclosure and Caged group macaques: Clear differences in the growth of body parts between macaques born in an enclosure and those born in cages were evident; many somatometric data of the Caged group were significantly smaller than those of the Enclosure group. How these differences come about became clear when we examined the growth of Japanese macaques at infantile and pubertal stages. Hamada analyzed the characteristics of 16 somatometric body measurements including body weight in the Japanese macaque [3]. His results showed that the growth curve for each somatometric item has a common 'bi-phasic' characteristic: growth consists of early or infantile growth and pubertal growth. The growth of many primates, including humans and other macaques, exhibits this 'bi-phasic' tendency $[15,17]$ which might account for the pattern of differences evident in this study. Similar trends of growth in several body parts, including head, face, and trunk related items in males and trunk related items in females, are thought to be due to identical rates of growth at the infantile and pubertal stages of the Enclosure and Caged groups. Differences in the growth of most head and face related items in females, and some girth related items in both sexes might be due to differences in growth mainly at the pubertal and infantile stages, respectively. Differences in some limb and girth related items might be due to growth rate differences both at the infantile and pubertal stages.

We believe that growth differences between the two groups were generated by differences in the rearing conditions. Although some exceptions exist, the somatometric data of the Caged group macaques were the same or lower than those of the Enclosure group macaques at every stage, suggesting that rearing in cages inhibited the innate growth of the animals. The growth of girths and chest breadth in both sexes and chest depth in males might be suppressed at the infantile stage when macaques are reared in cages. It is known in the Japanese macaque $[1,3]$, that a body part smaller in relative size to the adult at birth exhibits a high increment of growth in the first year of life, whereas a relatively larger part shows less incremental growth. Since body parts such as chest breadth, thigh girth and leg girth, are high or intermediate increment items at the infantile stage, rearing space can affect the growth of these measures at the infantile stage or in the first year of life.

The growth of some body parts was lower in the Caged group than in the Enclosure group macaques at every stage of development. These parts include hand length and breadth, foot length and breadth, maximal forearm girth and minimal leg girth in both sexes, minimal forearm girth and maximal leg girth in males, and head length and chest girth in females. The growth of these measures might have been suppressed by rearing in cages at the infantile stage and compensatory growth at the pubertal stage is thought to have occurred incompletely when the animals were reared continuously in cages. Although the average values of most measurements of the Caged group were smaller than those of the Enclosure group, some measurements, such as bigonial breadth, sitting height, anterior trunk length, bitrochanteric breadth, upper arm length in males, and bi-iliac breadth in both sexes, were larger in the Caged group animals. In males, differences in growth of bigonial breadth, bi-iliac breadth, bitrochanteric breadth, sitting height and anterior trunk length appeared and disappeared at the pubertal stage. These results suggest that growth of these 5 measured items in the Caged group accelerated at the pubertal stage. We can suggest no plausible reason at present for either accelerated growth rates or for larger mean values of upper arm length and bi-iliac breadth in the Caged group.

Sex differences in the appearance of growth differences : Differences in growth patterns between the sexes were most obvious in head, face and trunk related items. Sexual dimorphism in macaques is pronounced [9] and, in particular, differences between the sexes in size and growth of the head and face are considerable [14]. One important factor affecting growth of the head and facial elements is development of the masticatory apparatus, i.e., the eruption of deciduous and permanent teeth and the development of the muscles of mastication. The masticatory apparatus is thought to be more important for males than for females because it is essential for fighting enemies. Strong selection pressure for proper growth of the masticatory apparatus might operate to ensure stable innate growth of head and facial elements in males of both the Enclosure and Caged groups. In males, some trunk related items exhibited greater acceleration of growth at the pubertal stage in the Caged group. Caged group females, however, exhibited no such trend.

Effects of locomotion: Growth in the length and breadth of both hands and feet differed significantly between the Enclosure and Caged groups at the infantile and pubertal 
stages in both sexes. These measures are important not only in terms of locomotion but also for grasping the mother's belly or back in newborns. Hamada has reported that characteristics of postnatal growth imply either: (1) that the macaque grows up not to be a cursorial or saltatorial specialist but to be a plantigrade quadruped and/or climber, or (2) that the body of the newborn macaque is so well developed (i.e. relatively strong feet and hands) as to give the baby the ability to grip firmly [2, 13]. As the main difference between an enclosure and cage is space, macaques born in an enclosure might locomote more so than those born in a cage. This difference may consequently affect the growth of locomotion elements. Infants in a cage may also have less need to grasp the mothers belly or back because she has less space in which to move. Growth in wrist and ankle girths, which differed significantly between the Enclosure and Caged groups at the infantile and pubertal stages, are also important elements for locomotion.

The effect of feeding : Although food and water were supplied in the same amount to the Enclosure and Caged groups, the amount of intake of food and water might vary between these groups. In the Enclosure group, the social rank of juvenile and young macaques is lower than that of adults, so they may not be able to take as much food as they need until they are fully grown. In contrast, Caged group macaques experience little or no food competition that may confer on them an advantage over Enclosure group animals in terms of food intake. However, many somatometric measures of the Enclosure group were larger than or similar to those of Caged group macaques. Therefore, the growth differences detected probably do not reflect to any degree of a difference in food intake.

The present study revealed that the growth measurements with a large growth increment are more sensitive to differences in rearing conditions at the infantile growth stage in both sexes. Many measurements that exhibited retardation at the infantile stage recovered from the delay in growth at the pubertal growth stage. However, the size of some locomotor elements (e.g., the wrist and hand, or ankle and foot) strongly reflected limitations of space and changes due to this were irreversible. Further, our results suggest that females are more sensitive than males to space restrictions during growth. Ample rearing space and/or no height limitation is recommended for innate growth to occur in this species.

ACKNOWLEDGMENTS. We are grateful to Prof. Y. Hayashi, Graduate School of Agricultural and Life Sciences, The University of Tokyo, Tokyo, Japan and Prof. T. Kageyama, Primate Research Institute of Kyoto University, Aichi, Japan for their valuable advice. We thank Ms. Y. Suzuki for her technical assistance.

\section{REFERENCES}

1. Hamada, Y. 1982. Longitudinal somatometrical study on the growth patterns of newborn Japanese monkeys. Primates 23: 542-557.

2. Hamada, Y. 1983. Growth of the locomotor apparatus of the Japanese macaque: Based on analysis of muscular weights and limb segment lengths. J. Anthrop. Soc. Nippon 91: 347-362.

3. Hamada, Y. 1994. Standard growth patterns and variations in growth patterns of the Japanese monkeys (Macaca fuscata) based on an analysis by the spline function method. Anthropol. Sci. 102 (Suppl.): 57-76.

4. Hamada, Y., Watanabe, T. and Iwamoto, M. 1996. Morphological variations among local populations of Japanese macaque (Macaca fuscata). pp. 97-115. In: Variations in the Asian Macaques (Shotake, T. and Wada, K. eds.), Tokai University Press, Tokyo.

5. Ikeda, J. and Hayama, S. 1964. Body measurements of wild Japanese monkeys. pp. 93-108. In: Takasakiyama no Saru (Wild Japanese Monkeys in Takasakiyama) (Itani, J., Ikeda, J. and Tanaka, T. eds.), Keiso-shobo, Tokyo.

6. Iwamoto, M. 1971. Morphological studies of Macaca fuscata (VI): Somatometry. Primates 12: 151-174.

7. Martin, R. and Saller, K. 1957. Lehrbuch der Anthropologie. 3, Gustav Fischer Verlag, Stuttgart.

8. Matsubayashi, K. and Enomoto, T. 1983. Longitudinal studies on annual changes in plasma testosterone, body weight and spermatogenesis in adult Japanese monkeys (Macaca fuscata fuscata) under laboratory conditions. Primates 24: 521-529.

9. Napier, J. R. and Napier, P. H. 1967. A handbook of Living Primates. Academic Press, London.

10. Nigi, H., Tiba, T., Yamamoto, S., Floescheim, Y. and Ohsawa, N. 1980. Sexual maturation and seasonal changes in reproductive phenomena of male Japanese monkeys (Macaca fuscata) at Takasakiyama. Primates 21: 230-240.

11. Nozaki, M. 1993. Reproductive characteristics of Japanese monkeys. J. Reprod. Dev. 39: 93-107.

12. Ota, K., Makino, Y., Kimura, M. and Suzuki, J. 1991. Lactation in the Japanese monkey (Macaca fuscata): Yield and composition of milk and nipple preference of young. Primates 32: $35-48$.

13. Shimizu, T., Ono, T., Yoshida, T., Cho, F. and Goto, N. 1988. Morphometrical study of physical growth in infant cynomolgus monkeys using multivariate analysis. Exp. Anim. 37: 145-151.

14. Sirianni, J. E. 1985. Nonhuman primates as models for human craniofacial growth. pp. 95-124. In: Nonhuman Primate Models for Human Growth and Development (Watts, E. S. ed.), Alan R. Liss, Inc., New York.

15. Tanner, J. M. 1962. Growth at Adlescence. 2nd ed., Blackwell Scientific Publications, Oxford.

16. Watanabe, T. 1975. Some features of the wild Japanese monkeys (Macaca fuscata) of Shiga-A troop from viewpoint of somatometry. Phys. Ecol. 16: 55-63.

17. Watts, E. S. 1985. Adolescent growth and development of monkeys, apes and humans. pp. 41-65. In: Nonhuman Primate Models for Human Growth and Development (Watts, E. S. ed.), Alan R. Liss, Inc., New York. 